\title{
制备蛋白 $\mathrm{A}$ 单分子膜层装配抗体构建肿幦标志物 检测芯片
}

黄承洪 ${ }^{(1)}$, 王春霞 ${ }^{(2)}$ ，朱伟 ${ }^{(3)}$ ，侯长军 ${ }^{(1)}$ ，霍丹群 ${ }^{(1)^{*}}$, 陈艳艳 ${ }^{\left({ }^{*}\right.}$ ，靳刚 ${ }^{(4 *}$

(1) 重庆大学教育部生物流变学重点实验室, 重庆 400044;

(2) 中国科学院苏州纳米技术与纳米仿生研究所, 苏州 215123;

(3) 山东省医学科学院放射医学研究所, 济南 250062;

(4) 中国科学院力学研究所, 北京 100190

* 联系人, E-mail: huodq@cqu.edu.cn; yychen2006@sinano.ac.cn; gajin@imech.ac.cn

2012-05-07 收稿, 2012-07-02 接受, 2012-11-06 网络版发表

国家科技支撑计划(2012BAI19B03)、国家自然科学基金(81171414, 31171684, 11274345)、中国博士后科学基金(2011M500143)、高等学校 博士学科点专项科研基金(20090191110030)和重庆大学大型仪器设备开放基金资助

摘要 免疫分析方法主要依赖于固定在固相基底上的抗体对相应抗原的特异性识别, 抗体在固相 基底上固定后最大程度保持生物学活性是设计免疫分析方法的关键技术. 本研究利用在疏水性硅 基底表面制备蛋白 $\mathrm{A}$ 单分子膜层可以特异结合抗体的 Fc 端，进而实现对抗体的定向化装配，进一 步构建格式化阵列用于肿瘤标志物检测。研究结果表明, 制备的蛋白 $\mathrm{A}$ 单分子膜层的厚度为 $1.8 \pm 0.6 \mathrm{~nm}$, 抗体可以经蛋白 $\mathrm{A}$ 单分子膜层实现定向化装配, 由此设计的传感阵列检测肿瘤标志物

关键词

蛋白 $\mathrm{A}$

单分子层

成像椭偏

甲胎蛋白 甲胎蛋白 (alpha-fetoprotein, AFP) 可以实现 $1.0 \mathrm{ng} / \mathrm{mL}$ 的灵敏度, 血清检测结果与电化学法(ECLIA) 测定进行统计学检验没有显著性差异 $(P>0.05)$.

免疫分析已成为生物科学和生物医学最重要的 分析方法之一，免疫分析主要依赖于固定在固相基 底上的抗体对相应抗原的特异性识别而实现，要求 抗体在基底上固定应该最大程度地保持其固有的分 子结构和生物学活性, 这是设计新型免疫分析方法 的关键技术. 常规的抗体固定方法是经物理吸附或 者化学固定来实现, 由于物理吸附结合不牢固抗体 容易脱落，化学固定易致抗体活性损伤，因此设计一 种柔性有机膜层对抗体进行装配, 既可以保持抗体 的生物学活性又可以降低无关蛋白在基底表面的非 特异性吸附, 具有重要的理论和应用价值. 在固相基 底上制备单分子膜层对抗体定向化固定可以实现对 抗原的敏感性检测，有很多方法可以实现．例如，蛋 白 $\mathrm{A}$ 可以结合抗体的 $\mathrm{Fc}$ 端使抗体的 $\mathrm{Fab}$ 端朝外有利
于抗原结合 ${ }^{[1]}$; 氧化抗体 $\mathrm{Fc}$ 端的糖链结构形成开环 的醛基可以与改性基底的功能基团共价连接实现抗 体的定向化固定 ${ }^{[2]}$; 用还原剂使抗体的颈基开链实现 在金基底上对抗体定向化固定 ${ }^{[3]}$; 还可以加工有机物 膜垫 ${ }^{[4]}$ 或者磷脂膜层 ${ }^{[5]}$, 通过膜垫上的功能分子实现 对抗体的共价连接. 后面两种方法操作条件复杂苛 刻, 应用受到限制. 本文采用制备蛋白 $\mathrm{A}$ 单分子膜层 实现对抗体的定向化装配, 并构建蛋白质阵列实现 肿瘤标志物的无标记高灵敏检测.

\section{1 材料与方法}

（i ）试剂. 抛光硅片购自北京有色金属研究院; 鼠抗甲胎蛋白单克隆抗体(anti-AFP)购自中国原子能 科学研究院; AFP 标准品购买自中国药品生物制品检

引用格式: 黄承洪, 王春霞, 朱伟, 等. 制备蛋白 A 单分子膜层装配抗体构建肿瘤标志物检测芯片. 科学通报, 2013, 58: 575-579

Huang C H, Wang C X, Zhu W, et al. Preparation of single molecular film of protein A to assemble antibody for construction of biomarker chip (in Chinese). Chin Sci Bull (Chin Ver), 2013, 58: 575-579, doi: 10.1360/972012-701 
定所; 牛血清白蛋白(BSA)和蛋白 A 以及二氯二甲基 硅烷购自 Sigma公司; 肝癌病人血清来自山东省医学 科学研究院; 电化学分析试剂盒为商业试剂盒 (Roche); 去离子水 $(18 \mathrm{M} \Omega \mathrm{cm})$ 由 Millipore-Milli $\mathrm{Q}$ 纯 化系统纯化; 其余化学试剂均为分析纯, 购自国药集 团化学试剂公司.

(ii) 椭偏光学成像系统. 椭偏光学成像技术是 一种超薄膜及表面显示技术, 它将传统的光学椭偏 术和 CCD 成像技术、计算机采样技术及数字图像处 理技术相结合, 对薄膜的厚度分辨率可达原子层量 级, 横向分辨可达微米量级, 并具有大面积成像测 量、采样速度快和结果直观等优点, 可以定量显示生 物分子膜层的分布及其变化, 实现对蛋白质检测的 直接观察 ${ }^{[6]}$. 其原理见图 1, 由光源(light source)、起 偏器 (polarizer)、补偿器 (compensator)、检偏器 (analyzer) 和探测器 (detector) 等部分构成. 它以偏振 光波为探测光照射样品 (sample), 样品会对人射光波 进行调制, 使得反射光波中载有样品的信息, 经 CCD 摄像机探测, 结果以数字图像形式采集和存储, 便于分析和传输. 利用这种高灵敏度光学技术可以 实现对样品的无接触、无扰动、无标记检测 ${ }^{[7]}$. 结合 基底化学改性处理, 对基底进行表面格式化, 在 1.0 $\mathrm{cm} \times 1.5 \mathrm{~cm}$ 基底上可以实现 24 48 单元点, 即 24 48 个样品的通量检测 ${ }^{[8]}$.

(iii) 硅片改性. 硅片在 $\mathrm{H}_{2} \mathrm{SO}_{4} / \mathrm{H}_{2} \mathrm{O}_{2}(v / v=3: 1)$ 溶 液中氧化 $30 \mathrm{~min}$, 去离子水清洗 3 次得到氧化硅片. 被氧化的硅片在三氯乙烯/二氯二甲基硅烷 ( $v / v=20: 3)$ 混合溶液中反应 $5 \mathrm{~min}$, 先用三氯乙烯清洗 3 次, 然 后用乙醇清洗 3 次后再用去离子水冲洗, 氮气吹干后 得到疏水基底, 置冰箱 $4^{\circ} \mathrm{C}$ 保存备用.

(iv) 蛋白 $\mathrm{A}$ 单分子膜层装配. 浓度为 $1.0 \mathrm{~g} / \mathrm{mL}$

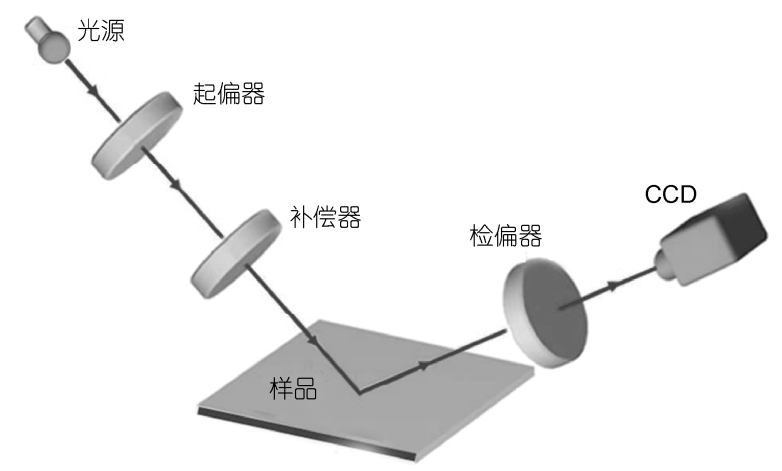

图 1 椭偏光学成像示意图
蛋白 $\mathrm{A}$ 溶解在 $\mathrm{PBS}(\mathrm{pH}$ 7.2)中, 不同稀释比例的蛋白 $\mathrm{A}$ 溶液经微流道加样系统以 $1.0 \mu \mathrm{L} / \mathrm{min}$ 的流速流经疏 水基底, 在基底表面格式化, 以阵列形式形成规则排 列的蛋白 $\mathrm{A}$ 分子膜层, 先用 PBS 清洗再用去离子水 清洗, 然后氮气吹干得到具有不同厚度的蛋白 $\mathrm{A}$ 分 子膜层.

(V) 抗体固定与抗原检测. 浓度为 $100.0 \mu \mathrm{g} / \mathrm{mL}$ 的 anti-AFP 经微流道加样系统以 $1.0 \mu \mathrm{L} / \mathrm{min}$ 的流速 在格式化的蛋白 A 膜层组装 $30 \mathrm{~min}, \operatorname{PBST}(0.02 \%$ Tween-20)以 $10.0 \mu \mathrm{L} / \mathrm{min}$ 的流速清洗 $5 \mathrm{~min}$, 再用相 同流速的 PBST 清洗 $10 \mathrm{~min}$, 得到蛋白 $\mathrm{A} /$ 抗体传感膜 层; 最后用 $\mathrm{BSA}(1.0 \mathrm{mg} / \mathrm{mL})$ 封闭 $20 \mathrm{~min}$, PBST 以 $10.0 \mu \mathrm{L} / \mathrm{min}$ 的流速清洗 $5 \mathrm{~min}$, 形成检测 AFP 的传感 膜层, 操作均在常温进行. 不同浓度 AFP 溶液分别 流过不同的传感膜层 $(1.0 \mu \mathrm{L} / \mathrm{min})$ 进行抗原抗体反应, PBS 清洗 $5 \min$ (流速 $10 \mu \mathrm{L} / \mathrm{min}$ )后, 再用清水冲洗 3 次后氮气吹干, 椭偏成像测定.

\section{2 结果与讨论}

\section{1 蛋白 $\mathrm{A}$ 单分子膜层}

经浓硫酸/双氧水清洗的硅片接触角为 $5^{\circ} \pm 1.0^{\circ}$, 再经二氯二甲基硅烷处理后的接触角为 $86^{\circ} \pm 2.1^{\circ}$, 具 有较高的疏水性. 蛋白质在水溶液中呈外部亲水性、 内部疏水性的球体或者椭球体 ${ }^{[9]}$, 肽链中疏水性氨基 酸对蛋白质在化学表面的吸附有直接影响, 如 Marsh 等人 ${ }^{[10]}$ 用光学椭偏和傅里叶红外研究了近似单分子 膜层的 $\beta$-乳球蛋白在亲/疏水性表面的吸附, 结果表 明在疏水性表面的吸附数量远比在亲水性表面的多, 且在疏水性表吸附更加牢固. 由于这种疏水作用力 直接影响蛋白质的结构稳定性和生物学活性 ${ }^{[11]}$, 因 此对于抗体直接在疏水性表面固定并不合适. 但是, 可以通过制备对抗体具有特异性结合能力的蛋白 A 单分子层实现对抗体进行定向化装配. 我们配制浓 度分别为 5.0, 10.0, 50.0, 100.0, 200.0, 400.0 和 600.0 $\mu \mathrm{g} / \mathrm{mL}$ 的蛋白 $\mathrm{A}$ 溶液在疏水性表面吸附 $10 \mathrm{~min}$, 随着 蛋白 $\mathrm{A}$ 浓度的增加其分子膜层的厚度(灰度值)也 逐渐增加, 由于膜层厚度 $(d)$ 正比于反射光强 $(I)$ 的平 方根 ${ }^{[12]}$ :

$$
I=k d^{2},
$$

其中 $k$ 为常数, 其数值可以通过已知厚度的蛋白质膜 层来确定. 也可以用蛋白质吸附的面密度来表征, 蛋 
白质膜层的面密度 (surface concentration, $\Gamma$ ) 和厚度之 间存在如下关系 ${ }^{[13]}$ :

$$
\Gamma\left(\mu \mathrm{g} / \mathrm{cm}^{2}\right) \approx K \times d(\mathrm{~nm}),
$$

其中 $K$ 为常数, 当基底为硅时, $K \approx 0.12$. 因此, 可以 得到蛋白 $\mathrm{A}$ 分子膜层的吸附厚度与浓度之间的函数 关系(图 2), 随蛋白 $\mathrm{A}$ 浓度的增加, 蛋白 $\mathrm{A}$ 膜层的面 密度也相应增加, 当蛋白 $\mathrm{A}$ 浓度达到 $200.0 \mu \mathrm{g} / \mathrm{mL}$ 时 的分子面密度处于临界值, 根据换算其膜层厚度为 $1.8 \pm 0.6 \mathrm{~nm}$. 由于蛋白分子的分子量为 $45000 \mathrm{Da}$ 左右, 其干膜理论厚度(粗偏测量)接近 $2.0 \mathrm{~nm}^{[5]}$, 因此我们 认为 $200.0 \mu \mathrm{g} / \mathrm{mL}$ 浓度时得到单分子膜层. 装配单分 子膜层对于抗体进行固定具有重要的意义, 如果蛋 白 $\mathrm{A}$ 的浓度过小, 在疏水表面形成的分子膜层有较 大的空隙, 会导致无关蛋白吸附本底增高, 浓度过大, 形成的分子膜层是多层结构, 抗体结合后在清洗步 骤容易脱落, 导致假阴性. 我们把疏水性表面和 $200.0 \mu \mathrm{g} / \mathrm{mL}$ 蛋白 A 吸附膜层进行原子力观察, 如图 3 所示, 疏水表面具有很好的平整度, 当吸附蛋白 $\mathrm{A}$ 后, 表面粗鋉度增加, 截面分析与椭偏厚度测定一致, 进一步证实了得到的分子膜层是单分子膜层.

\section{2 抗体在蛋白 $\mathbf{A}$ 膜层上装配}

经过优化确定 $200.0 \mu \mathrm{g} / \mathrm{mL}$ 蛋白 $\mathrm{A}$ 为工作浓度, anti-AFP 分别以 20.0, 40.0, 60.0, 80.0, 100.0, 120.0 和 $140.0 \mu \mathrm{g} / \mathrm{mL}$ 与制备的蛋白 A 分子膜层进行特异性结 合. 同样, 随 anti-AFP 浓度的增加其蛋白质单元点的 灰度也随之增加(图 4), 当浓度超过 $100.0 \mu \mathrm{g} / \mathrm{mL}$ 时灰 度增加不明显, 说明在基底表面吸附的蛋白 $\mathrm{A}$ 的活 性位点随抗体的浓度增大被逐渐占用, $100.0 \mu \mathrm{g} / \mathrm{mL}$

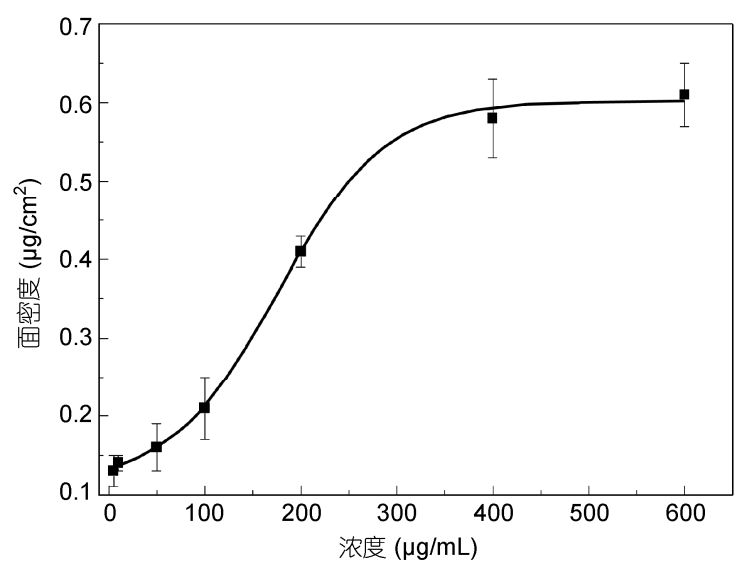

图 2 蛋白 $\mathrm{A}$ 浓度与吸附分子膜层的函数关系

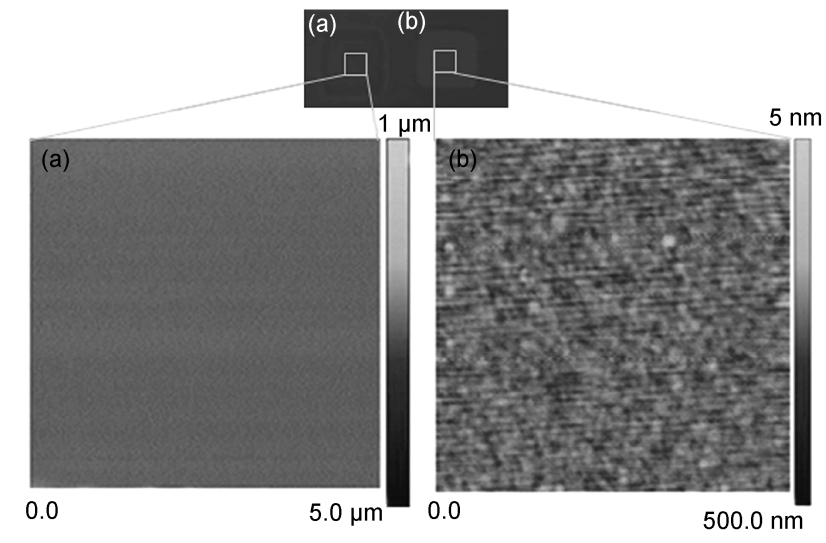

图 3 疏水基底表面(a)和蛋白 $\mathbf{A}$ 在疏水表面的单分子膜层 原子力 AFM 图像(b)

浓度时达到饱和, 形成的分子膜层(传感膜层)可用于 抗原检测.

\section{3 抗原结合活性及血清应用}

为了验证制备的蛋白 $\mathrm{A}$ 单分子膜层装配抗体的 实用价值, 我们对传感膜层的应用潜能进行了研究. 如图 5 所示, 构建的阵列采取 $4 \times 4$ 排列, 第一行中的 4 个单元点作为对照点, 即单元点 $a_{1}, b_{1}, c_{1}$ 为传感膜 层结合 $25.0 \mathrm{ng} / \mathrm{mL}$ 抗原 (3 个重复), 平均灰度值为 $96 \pm 4.6$, 单元点 $d_{1}$ 作为空白对照, 灰度值为 85.8. 其余单元点 $\left(\mathrm{a}_{2} \sim \mathrm{d}_{4}\right)$ 用于血清检测, 血清样品以 1.0 $\mu \mathrm{L} / \mathrm{min}$ 流速与传感膜层反应 $20 \mathrm{~min}$, 然后 $\mathrm{PBS}$ 以 $10.0 \mu \mathrm{L} / \mathrm{min}$ 流速清洗 $5 \mathrm{~min}$, 氮气吹干, 经椭偏成像 后显示出不同的灰度值. 根据其净灰度值高出对照

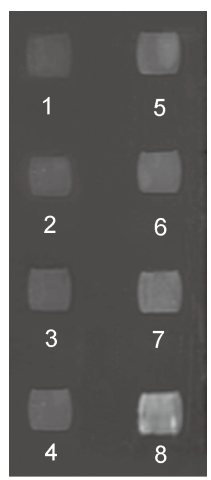

图 4 Anti-AFP 在蛋白 A 分子膜层上装配, 随 anti-AFP 浓度 的增加其结合数量越多, 椭偏图像上各单元点的灰度值增加, $1 \sim 8$ 单元点的灰度值分别为 $56.4,62.2,69.8,86.5,94.6,101.4$, 110.6, 118.2 


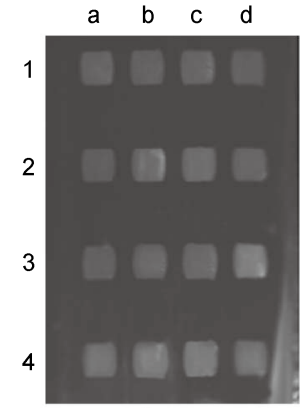

(A)

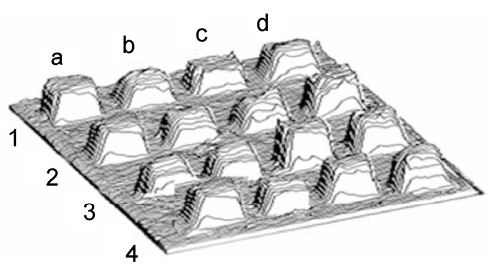

(B)
图 5 临床样本测定结果

(A) 灰度图; (B) 三维图. 单元点 $\mathrm{a}_{1} \sim \mathrm{a}_{3}$ 为阳性对照 $(25.0 \mathrm{ng} / \mathrm{mL}$, 3 个重复), 单元点 $b_{1}$ 作为空白对照; 单元点 $a_{2} \sim d_{4}$ 用于血清检测

3 倍标准差判定 $a_{4}, b_{2}, b_{4}, c_{2}$ 和 $d_{3}$ 为阳性. 此外, 我们 还制定了不同抗原浓度反应后的标准曲线，可以用 回归方程式 $y=-29.3+93.6 \log x\left(R^{2}=0.989\right)$ 进行描述.
根据计算, 检测灵敏度达到 $1.0 \mathrm{ng} / \mathrm{mL}(\mathrm{S} / \mathrm{N}=3)$, 测定 $25.0 \mathrm{ng} / \mathrm{mL}$ 甲胎蛋白的重现性 $(\mathrm{CV} \%)$ 为 $10.4 \%$. 本方 法与电化学法(ECLIA)测定结果进行统计学检验, 没 有显著性差异 $(P>0.05)$. 由于该方法简单快速，可望 用于肝癌血清标志物(AFP)大批量篎查.

\section{3 结论}

以蛋白 $\mathrm{A}$ 在疏水性硅基底表面吸附为研究对象, 不同浓度蛋白 $\mathrm{A}$ 在疏水性表面吸附后经成像椭偏表征, 证实 $200.0 \mu \mathrm{g} / \mathrm{mL}$ 浓度得到单分子膜层, 厚度为 $1.8 \pm$ $0.6 \mathrm{~nm}$, 该膜层可以对甲胎蛋白抗体实现定向化固定. 优化抗体的最适装配浓度为 $100.0 \mu \mathrm{g} / \mathrm{mL}$, 用于制备 传感膜层构建传感阵列. 构建的传感阵列对甲胎蛋白 的检测灵敏度达到 $1.0 \mathrm{ng} / \mathrm{mL} ，$ 血清检测结果与电化 学法进行统计学检验没有显著性差异 $(P>0.05)$.

\section{参考文献}

1 Wang Z, Jin G. Feasibility of protein A for the oriented immobilization of immunoglobulin on silicon surface for a biosensor with imaging ellipsometry. J Biochem Biophys Methods, 2003, 57: 203-211

2 Yuan Y, Yin M, Qian J C, et al. Site-directed immobilization of antibodies onto blood contacting grafts for enhanced endothelial cell adhesion and proliferation. Soft Matter, 2011, 7: 7207-7216

3 Karyakin A A, Presnova G V, Yu M Y, et al. Oriented immobilization of antibodies onto the gold surfaces via their native thiol groups. Anal Chem, 2000, 72: 3805-3811

4 Ordonez S S, Fabregas E. New antibodies immobilization system into a graphite-polysulfone membrane for amperometric immunosensors. Biosens Bioelectron, 2007, 22: 965-972

5 Zhang Y, Chen Y, Jin G. PEGylated phospholipid membrane on polymer cushion and its interaction with cholesterol. Langmuir, 2010, 26: $11140-11144$

6 Nygren H, Sandström T, Stenberg M. Direct visual detection of protein antigen: Importance of surface concentration. J Immunol Methods, 1983, 59: 145-149

7 Schram T, Franquet A, Terryn H, et al. Spectroscopic ellipsometry: A non-destructive technique for surface analysis. Adv Eng Mater, 2000, 1: 63-66

8 Wang Z H, Jin G. A label-free multisensing immunosensor based on imaging ellipsometry. Anal Chem, 2003, 75: 6119-6123

9 Tisi L C, Evans P A. Conserved structural features on protein surfaces: Small exterior clusters. J Mol Biol, 1995, 249: 251-258

10 Marsh R J, Jones R A L, Sferrazza M. Adsorption and displacement of a globular protein on hydrophilic and hydrophobic surfaces. Coll Surf B: Biointerf, 2002, 23: 31-42

11 Pace C N. Contribution of hydrophobic interactions to protein stability. J Mol Biol, 2011, 408: 514-528

12 Arwin H, Lundstrgm I. A reflectance method for quantification of immunological reactions on surfaces. Anal Biochem, 1985, 145: $106-112$

13 Chen Y, Jin G. Refractive index and thickness analysis of natural silicon dioxide film growing on silicon with variable-angle spectroscopic ellipsmetry. Spectroscopy, 2007, 21: 26-31 


\title{
Preparation of single molecular film of protein A to assemble antibody for construction of biomarker chip
}

\author{
HUANG ChengHong ${ }^{1}$, WANG ChunXia ${ }^{2}$, ZHU Wei $^{3}$, HOU ChangJun ${ }^{1}$, HUO DanQun ${ }^{1}$, \\ CHEN YanYan ${ }^{2}$ \& JIN Gang ${ }^{4}$ \\ ${ }^{1}$ Key Laboratory of Biorheological Science and Technology, Ministry of Education, Bioengineering College, Chongqing University, Chongqing \\ 400044, China; \\ ${ }^{2}$ Suzhou Institute of Nano-tech and Nano-bionics, Chinese Academy of Sciences, Suzhou 215123, China; \\ ${ }^{3}$ The Radiation Medical Institute, Shandong Academy of Medical Sciences, Jinan 250062, China, \\ ${ }^{4}$ Institute of Mechanics, Chinese Academy of Sciences, Beijing 100190, China
}

Immunoassay is mainly performed by the immobilized antibody on solid substrate to specifically recognize the corresponding antigen. The key technique is the immobilization of antibody on solid substrate to preserve its biological activities. Here we utilize the protein A to prepare single molecular film on hydrophobic silicon substrate, which can realize site-directed immobilization of antibody by the combination of its Fc fragment for biomarker detection through constructing array format. The results showed that the thickness of single molecular film of protein A measured by imaging ellipsometry is approximately $1.8 \pm 0.6 \mathrm{~nm}$ and it could be used for sitedirected immobilization of antibody. The designed sensing array for AFP detection possesses high sensitivity of $1.0 \mathrm{ng} / \mathrm{mL}$. No statistical difference is found $(P>0.05)$ compared with that of ECLIA.

protein A, single molecular film, imaging ellipsometry, a-fetoprotein

doi: 10.1360/972012-701 